\title{
miR-204 regulates the biological behavior of breast cancer MCF-7 cells by directly targeting FOXA1
}

\author{
SI-QIAO SHEN ${ }^{*}$, LAN-SHAN HUANG ${ }^{*}$, XIAO-LING XIAO, XIAO-FEI ZHU, DAN-DAN XIONG, \\ XUE-MEI CAO, KANG-LAI WEI, GANG CHEN and ZHEN-BO FENG
}

Department of Pathology, The First Affiiated Hospital of Guangxi Medical University, Nanning, Guangxi 530021, P.R. China

Received October 14, 2016; Accepted November 30, 2016

DOI: $10.3892 /$ or.2017.5644

\begin{abstract}
MicroRNAs (miRNAs) are short, non-proteincoding RNAs and transcripts that are 18-24 nt in length. miR-204 was first identified as an anti-oncogene and is reported to be downregulated in non-small cell lung cancer, glioma, gastric and thyroid cancer. Recent studies have proposed that a low level of miR-204 expression is associated with tumor progression and disease outcome in breast cancer. Forkhead box A1 (FOXA1), a transcription factor, plays a crucial role in breast cancer and has been predicted as a target of miR-204. In the present study, we integrated the results of microarray analyses of breast cancer tissues obtained from an online database with our own determination of the expression of miR-204 in breast cancer MCF-7 cells using real-time qPCR (RT-qPCR). The proliferative capacity of the cells was assessed using MTT assays, and cell mobility and invasiveness were evaluated using cell migration and invasion assays, respectively. Flow cytometry was used to analyze apoptosis. FOXA1 levels were detected using RT-qPCR and western blot analysis. Luciferase assays were performed to confirm that FOXA1 is directly targeted by miR-204. The results showed that miR-204 was downregulated in breast cancer cells, and we found that miR-204 was expressed at a lower level in
\end{abstract}

Correspondence to: Professors Zhen-Bo Feng or Gang Chen, Department of Pathology, The First Affiliated Hospital of Guangxi Medical University, 6 Shuang Yong Road, Nanning, Guangxi 530021, P.R. China

E-mail: guanghu1963@126.com

E-mail: chen_gang_triones@163.com

${ }^{*}$ Contributed equally

Abbreviations: miRNAs, microRNAs; FOXA1, forkhead box A1; $\mathrm{HNF} 3 \alpha$, hepatocyte nuclear factor $3 \alpha$; TTR, transthyretin; $\alpha 1-\mathrm{AT}$, $\alpha 1$-antitrypsin; AR, androgen receptor; ESR1, estrogen receptor 1; NKX2-1, NK2 homeobox 1; INS, insulin; SCGB1A1, secretoglobin, family 1A, member 1; SHH, sonic Hedgehog; FOXA2, Forkhead box A2; TFF1, trefoil factor 1; NKX3-1, NK3 homeobox 1; ER, estrogen receptor

Key words: breast cancer, miR-204, FOXA1
MCF-7 cells than that observed in normal breast epithelial HBL-100 cells. Overexpression of miR-204 inhibited cell proliferation, migration and invasion and promoted apoptosis. Western blot analysis revealed that the expression of FOXA1 at the protein level was significantly reduced after cells were transfected with miR-204-expressing viruses. Luciferase assays demonstrated that FOXA1 is a direct target of miR-204, which binds to FOXA1 in a complementary region. In conclusion, miR-204 regulates the biological behavior of breast cancer cells, including cell proliferation, invasion, metastasis and apoptosis, by directly targeting FOXA1. Thus, miR-204 may act as a tumor-suppressor, and the results of the present study provide a reference for future research into the potential mechanisms underlying breast cancer progression.

\section{Introduction}

Breast cancer is the most common type of cancer in women worldwide, and it is also the leading cause of cancer-related deaths among women in both less developed countries and worldwide (1). Despite the advancements that have been made in multidisciplinary and diagnostic approaches, the incidence of breast cancer and the associated mortality rate remain as high as 25.1 and $14.7 \%$, respectively (2). In the US, breast cancer was the most frequently diagnosed cancer in women in 2016, with the proportion of such cancers reaching $29 \%$ (3). In China, the situation is even more severe since women younger than 45 years of age who are diagnosed with breast cancer generally succumb to the disease and the age-standardized incidence rates have seen a significant upward trend (4). Currently, specific, standard and independent prognostic factors have been established for breast cancer, including tumor size, pathological grade, clinical stage and the presence of lymph node metastases, as well as hormone receptor status. Although these parameters can reflect the biological characteristics of a tumor, the underlying pathogenesis of breast cancer tumors remains unclear. Furthermore, both genetic and environmental factors contribute to the complex etiology of breast cancer. It is therefore essential and urgent that researchers investigate the mechanisms underlying breast cancer pathologies.

MicroRNAs (miRNAs) are short, non-protein-coding RNAs and transcripts that are 18-24 nt in length. miRNAs regulate gene expression at the post-transcriptional level by binding to complementary sites in the 3'-untranslated regions 
(3'-UTRs) of their target mRNAs $(5,6)$. miRNAs play a vital role in breast cancer as they are reported to be involved in tumor progression, particularly in blocking apoptosis and promoting uncontrolled cell disruption (7). For example, miR-155, miR-373 and miR-520c have each been shown to be upregulated, and to act as oncogenic miRNAs in breast cancer (8-10), whereas miR-34, miR-200c and miR-205 have been shown to be downregulated and to act as tumor suppressors in breast cancer (11-14). Li et al (15) demonstrated that the expression of miR-204 was expressed at markedly lower levels in breast cancer tissues than in adjacent normal breast tissues. Wang et al (16) found that miR-204 targeted and suppressed JAK2 which prevented the activation of Bcl-2, STAT3 and survivin in breast cancer cells. As a single miRNA targets multiple genes, and other target genes of miR-204 have not yet been fully identified, there is a possibility that the role of miR-204 in breast cancer is achieved through its effects on other targets. The molecular mechanisms involved in the role of miR-204 therefore remain to be investigated.

Using predictive online databases, we determined that fork head box A1 (FOXA1) is a prospective target gene of miR-204. FOXA1 is a member of the FOX family of transcription factors and is also known as hepatocyte nuclear factor $3 \alpha$ (HNF3 $\alpha$ ). Initially, FOXA1 was identified in the liver and has been reported to be a transcriptional regulator of transthyretin (TTR) and $\alpha 1$-antitrypsin ( $\alpha 1-A T)$ (17). High levels of FOXA1 have been reported in lung cancer, thyroid carcinoma, esophageal squamous cell carcinoma and prostate cancer (18-21), in addition to breast cancer (22). Notably, the current literature shows that the expression of FOXA1 is inversely proportional to that of miR-204 in breast cancer; when FOXA1 is highly expressed, miR-204 is expressed at low levels. When the opposite expression pattern is observed, the effects of both factors on biological behavior are also opposing. For instance, miR-204 restrains cell growth and induces apoptosis (16), whereas FOXA1 promotes cell growth and inhibits apoptosis (18). We therefore hypothesized that there may be a relationship between FOXA1 expression and miR-204 levels.

In the present study, we investigated the biological function of miR-204 in breast cancer and explored the potential molecular mechanisms by which miR-204 targets FOXA1 in the breast cancer cell line MCF-7.

\section{Materials and methods}

Cell lines and cultures. The human breast cancer MCF-7 cell line and the human embryonic kidney HEK293T cell line were purchased from the Shanghai Institute of Cell Biology (Shanghai, China). The human breast epithelial cell line HBL-100 was purchased from Boster Biotech (Wuhan, China). The cells were cultured in Dulbecco's modified Eagle's medium (DMEM) with high glucose (Wisent, Nanjing, China) and supplemented with $15 \%$ fetal bovine serum (FBS; ExCell Bio, Shanghai, China), $1 \mathrm{mM}$ penicillin-streptomycin solution and $1 \mathrm{mM}$ L-glutamine in a humidified incubator at $37^{\circ} \mathrm{C}$ with $5 \% \mathrm{CO}_{2}$.

miRNA transfection. The experiment was divided into three groups: control (normal MCF-7 cells), NC (MCF-7 cells infected with a negative control virus), and LV-hsa-mir-204
(MCF-7 cells infected with the miR-204-up virus). miR-204-up viruses (LV-hsa-mir-204) with the following sequences were obtained from GeneGeme (Shanghai, China): hsa-mir-204-P1, GAGGATCCCCGGGTACCGGTCCTGATCATGTACCCA TAGG and hsa-mir-204-P2, CACACATTCCACAGGCTAG CTTATGGGACAGTTATGGGC. MCF-7 cells were seeded into 6-well plates at a density of $1 \times 10^{5}$ cells/well (in a $1 \mathrm{ml}$ volume of cell medium), and then cultured overnight before transfection. Lentiviruses were added to the MCF-7 cells to induce infection. Three days after infection, the levels of intracellular fluorescent markers were analyzed using a fluorescence microscope. The cells were then harvested to examine the changes in the expression of miR-204 and FOXA1 mRNA and protein.

Target prediction. The following online biological databases were used to predict the targets of miRNAs: microRNA. org (http://www.microrna.org/microrna/home.do), miRWalk (http://www.umm.uni-heidelberg.de/apps/zmf/mirwalk/), DIANA (http://diana.imis.athena-innovation.gr/DianaTools/), and TargetScan (http://www.targetscan.org/).

Data integration. The cBioPortal database (http://www.cbioportal.org/), which is a subsidiary database of The Cancer Genome Atlas (TCGA; http://cancergenome.nih.gov/), was used to select chips of invasive breast cancer and to analyze the relevant clinical parameters for miR-204 and FOXA1. The STRING database (http://string-db.org/) was used to analyze the interactions of FOXA1.

RNA isolation, quality control and RT-qPCR. Total RNA was extracted from cells in each of the three groups using an AxyPrep Multisource Total RNA Miniprep kit (Axygen, Suzhou, China). The concentration of total RNA in the samples was determined using a NanoDrop 2000 instrument (Thermo Fisher Scientific, Waltham, MA, USA). The samples were required to meet the following qualifications: $1.8<\mathrm{OD}_{260 / 280}<2.0$. For reverse transcription, we used a Thermo Fisher Scientific RevertAid First Strand cDNA synthesis kit (Thermo Fisher Scientific) and a miRcute miRNA First-Strand cDNA synthesis kit (TianGen, Beijing, China), each according to the manufacturer's instructions, to obtain cDNA. qPCR analysis was performed to determine FOXA1 and miR-204 levels using SYBR-Green Premix Ex Taq (Roche Life Science) and miRcute miRNA qPCR detection kits (SYBR-Green) (TianGen), respectively, in a LightCycler 480 (Roche, Basel, Switzerland). $\beta$-actin and U6 were used as internal controls, respectively. The following primers were used: $\beta$-actin (23) forward, 5'-GCACCACACCTTCTACAATGAGC-3' and reverse, 5'-GGATAGCACAGCCTGGATAGCAAC-3'; and FOXA1 (24) forward, 5'-AATCATTGCCATCGTGTG-3' and reverse, 5'-CGCGGCTTAAAATCTGGTAT-3'. The primers for miR-204 and U6 were purchased from TianGen, and the sequences were determined.

Cell viability assay. Cell viability was assessed using 3-(4,5-dimethylthiazol-2-yl)-2,5-diphenyltetrazolium bromide (MTT; Sigma-Aldrich, St. Louis, MO, USA). Cells in each of the three groups were seeded in 96-well plates in a volume of $0.2 \mathrm{ml}$ of medium/well resulting in a density of 3,000 cells/well. 
The cells were incubated at $37^{\circ} \mathrm{C}$ with $5 \% \mathrm{CO}_{2}$ for 24,48 , 72 and $96 \mathrm{~h}$. MTT $(20 \mu \mathrm{l})$ was added to each well, and the cells were incubated for $4 \mathrm{~h}$ at $37^{\circ} \mathrm{C}$. The cell culture medium was then removed, and $150 \mu 1$ of dimethyl sulfoxide (DMSO) (Sigma-Aldrich) was mixed into each well to stop the reaction. The solution was shaken for $10 \mathrm{~min}$, and the optical densitiy (OD) value was detected at $490 \mathrm{~nm}$ using a microplate reader (Scientific Multiskan FC; Thermo Fisher Scientific).

Cell invasion and migration assays. A Transwell chamber with $8-\mu \mathrm{m}$ pores (Corning Inc., Corning, New York, USA) was prepared for the invasion and migration assays. Samples of each of the three groups of cells were resuspended in $200 \mu \mathrm{l}$ of DMEM, and seeded in the upper chambers at a density of $1 \times 10^{5}$ cells/well with (for the invasion tests) or without (for the migration tests) Matrigel (BD Biosciences, Franklin Lakes, NJ, USA). DMEM (500 $\mu$ l) supplemented with 15\% FBS was added to the bottom chambers. After $24 \mathrm{~h}$ (migration assays) or $48 \mathrm{~h}$ (invasion assay), the cells in the top chambers were removed using cotton swabs. The chambers and the penetrated cells were fixed with $4 \%$ paraformaldehyde and stained with $0.1 \%$ crystal violet for $30 \mathrm{~min}$. The number of cells was observed under an inverted microscope at a magnification of x 200 .

Flow cytometric analysis. A PE Annexin V apoptosis detection kit I (BD Biosciences) was used to detect the apoptotic effects of miR-204 according to the manufacturer's protocol. Samples of cells from each of the three groups were washed with cold phosphate-buffered saline (PBS) twice, and then, resuspended in $1 \mathrm{X}$ binding buffer. The solution was then transferred to a 5-ml culture tube. PE Annexin V $(5 \mu \mathrm{l})$ and $5 \mu 1$ of 7-AAD were added to the tubes, and the reagents were then mixed with the cells. The cells were incubated for $15 \mathrm{~min}$ in the dark. Then, $400 \mu \mathrm{l}$ of $1 \mathrm{X}$ binding buffer was added to each tube and the rate of apoptosis was analyzed using flow cytometry after $1 \mathrm{~h}$.

Protein extraction and western blot analysis. Total proteins were extracted from the cells, and western blot analyses were performed as previously described (25). The cells were treated with $99 \mathrm{mM}$ radio immunoprecipitation assay (RIPA) buffer and $1 \mathrm{mM}$ phenylmethanesulfonyl fluoride (PMSF). The lysates were centrifuged at $12,000 \mathrm{xg}$ for $5 \mathrm{~min}$ and boiled for $7 \mathrm{~min}$. The proteins were subjected to $8 \%$ SDS-PAGE for $2 \mathrm{~h}$ at $70 \mathrm{~V}$, and the separated proteins were then transferred to polyvinylidene fluoride (PVDF) membranes (Millipore, Billerica, MA, USA) for $90 \mathrm{~min}$ at $100 \mathrm{~mA}$. The membrane was then incubated with FOXA1 antibodies (1:1,000; Abcam, Cambridge, MA, USA) and GAPDH antibodies (1:10,000; KangChen, Shanghai, China) overnight at $4^{\circ} \mathrm{C}$. Next, the membranes were incubated for $1.5 \mathrm{~h}$ with horseradish peroxidase-conjugated goat anti-rabbit $\operatorname{IgG}$ secondary antibodies (1:50,000; Santa Cruz Biotechnology, Santa Cruz, CA, USA). Finally, the membranes were analyzed using chemiluminescence detection methods with a Pierce ECL Western Blot Substrate (Thermo Fisher Scientific).

Luciferase assay. The interaction between the 3'-UTR of FOXA1 and miR-204 was analyzed using plasmids synthesized by Biosense Technologies (Guangzhou, China).
HEK293T cells were seeded in 24-well plates at a density of $1.5 \times 10^{5}$ cells/well and cultured for $24 \mathrm{~h}$. The plasmids were transfected into the HEK293T cells using Lipofectamine 2000 (Invitrogen, Carlsbad, CA, USA). Luciferase activity was tested by a Dual Luciferase Reporter Assay System (Promega, Madison, WI, USA) $(10,23)$.

Statistical analyses. All experiments were performed in triplicate. The data were analyzed using SPSS 20.0 software (SPSS, Inc., Chicago, IL, USA) and are expressed as the means \pm standard deviations (SD). One-way analysis of variance (ANOVA) tests were performed to analyzed the results across the three groups. Two-way ANOVA tests were used to analyzed the results of the growth curves. Student's t-test was used to determine differences between the control and LV-hsa-mir-204 group. The data related to clinicopathological parameters were analyzed using Chi-square or Fisher's exact test. A value of $\mathrm{P}<0.05$ was considered to indicate statistical significance.

\section{Results}

miR-204 target gene predictions. FOXA1, a transcription factor, was one of the 8,312 predicted targets of miR-204. All four databases (microRNA.org, miRWalk, DIANA and TargetScan) predicted that its expression was correlated with levels of miR-204. FOXA1 has been reported to play an essential role in cell proliferation (26), the cell cycle (27), DNA methylation (28) and tumor development and progression (21). We therefore focused on the relationship between miR-204 and FOXA1.

miR-204 is expressed at low levels and FOXA1 is expressed at high levels in breast cancer tissues. We obtained and collated 1,105 breast cancer chips from the cBioPortal database. Among these, 898 and 1,095 cases expressed miR-204 and FOXA1, respectively. In addition, 100 normal chips were selected. miR-204 was expressed at lower levels in breast cancer tissues than these levels in normal tissues $(\mathrm{P}<0.001$; Fig. $1 \mathrm{~A})$, and FOXA1 was expressed at higher levels in breast cancer tissues than these levels in normal tissues ( $\mathrm{P}<0.001$; Fig. 1B).

Association of miR-204 and FOXA1 with clinicopathological characteristics. Of the 1,105, 808 and 999 cases were screened to obtain complete clinical data related to the expression of miR-204 and FOXA1. The identified correlations between miR-204 and tumor characteristics are shown in Table I. The expression of miR-204 was strongly associated with lymph node stage $(\mathrm{P}=0.026)$, cancer metastasis stage $(\mathrm{P}<0.0001)$, progesterone receptor $(\mathrm{PR})$ status $(\mathrm{P}=0.044)$ and Her2 status $(\mathrm{P}=0.014)$. As shown in Table II, FOXA1 expression was significantly correlated with age $(\mathrm{P}<0.0001)$, lymph node stage $(\mathrm{P}=0.022)$, estrogen receptor $(\mathrm{ER})$ status $(\mathrm{P}<0.0001)$ and $\mathrm{PR}$ status $(\mathrm{P}<0.0001)$.

miR-204 is downregulated and FOXA1 is upregulated in breast cancer MCF-7 cells. We detected the expression of miR-204 and FOXA1 in MCF-7 cells. As shown in Fig. 1C, miR-204 was expressed at significantly lower levels in the MCF-7 cells than that in the normal human breast epithelial 
A
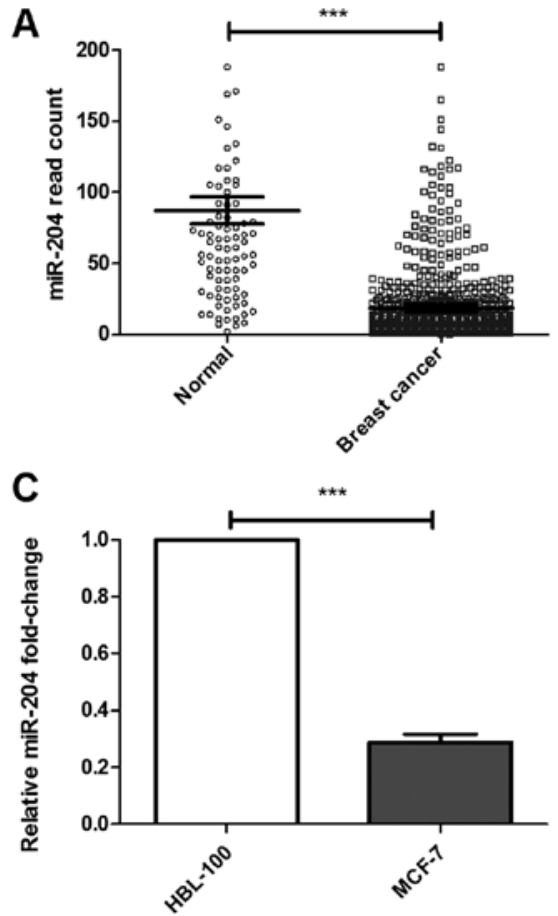

$\mathbf{E}$

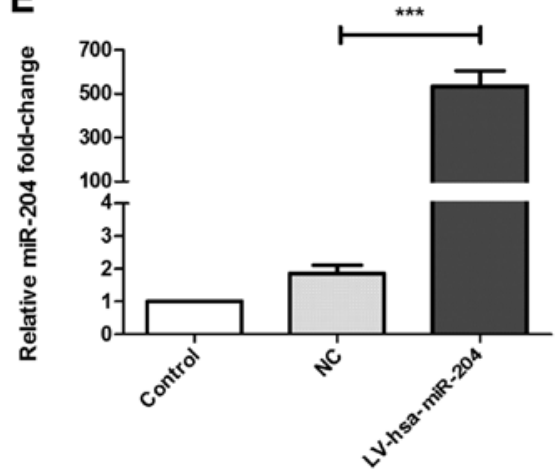

B
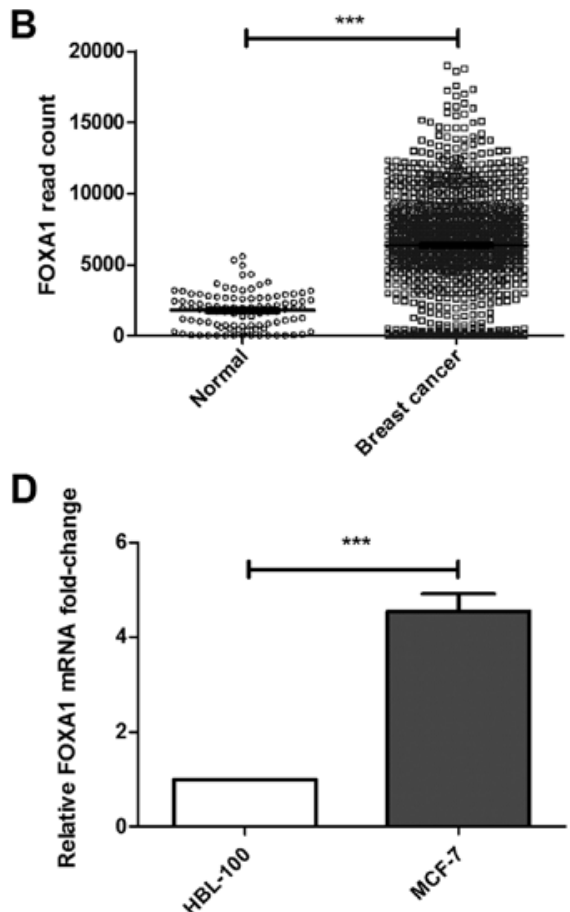

$\mathbf{F}$

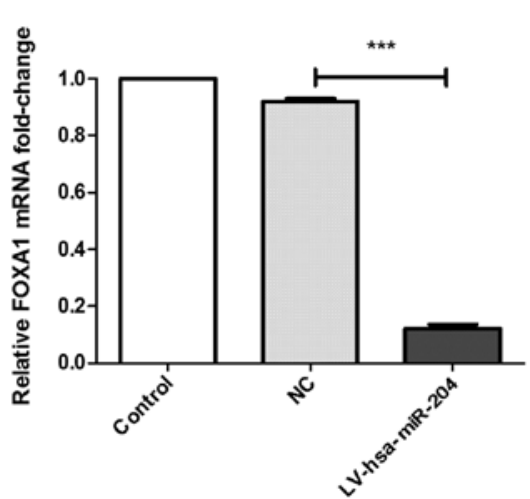

Figure 1. Levels of miR-204 and FOXA1 in breast cancer tissues and breast cancer MCF-7 cells. (A) miR-204 was expressed at lower levels in breast cancer tissues when compared with levels in normal breast tissues. (B) FOXA1 was expressed at higher levels in breast cancer tissues when compared with levels in normal breast tissues. (C) The expression of miR-204 was lower in MCF-7 cells when compared with that in HBL-100 cells. (D) The level of FOXA1 was higher in MCF-7 cells when compared with that in HBL-100 cells. (E) miR-204 was higher in the LV-hsa-mir-204 group compared with the control and NC groups. (F) FOXA1 mRNA was higher in the LV-hsa-mir-204 group than that noted in the control and NC groups; ${ }^{* * *} \mathrm{P}<0.001$.
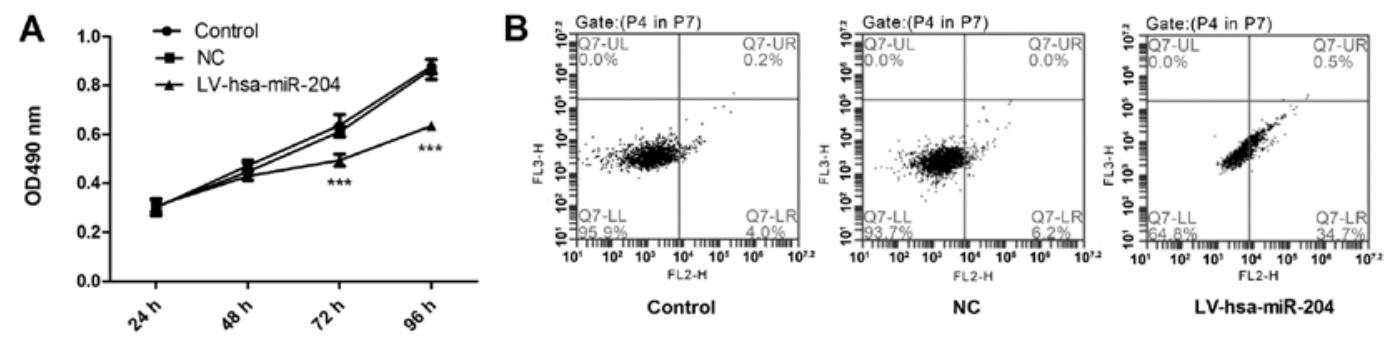

Figure 2. miR-204 inhibits proliferation and promotes apoptosis in breast cancer MCF-7 cells. (A) MCF-7 cells were transfected with miR-204-up. Proliferation was then monitored using MTT assays, and cell viability was markedly decreased at $96 \mathrm{~h}$ in the LV-hsa-mir-204 group. (B) Apoptosis was evaluated after transfection with miR-204-containing vector in three experimental groups using an Annexin V assay; ${ }^{* * *} \mathrm{P}<0.001$.

cells (HBL-100) $(\mathrm{P}<0.001)$. Meanwhile, FOXA1 was highly expressed in the MCF-7 cells $(\mathrm{P}<0.001$; Fig. 1D). To determine whether there is an interaction between miR-204 and FOXA1, we transfected the miR-204-up virus (LV-hsa-mir-204) into MCF-7 cells $(\mathrm{P}<0.001 ;$ Fig. 1E). The miR-204 mRNA level was markedly increased, while the FOXA1 mRNA level was strikingly decreased $(\mathrm{P}<0.001$; Fig. $1 \mathrm{~F})$.

miR-204 inhibits proliferation and induces apoptosis in MCF-7 cells. To determine the effect of upregulated levels of 
Table I. Associations between miR-204 expression and clinicopathological characteristics of the breast cancer cases $(\mathrm{N}=809)$.

\begin{tabular}{|c|c|c|c|c|}
\hline Characteristics & $\begin{array}{c}\text { No. of } \\
\text { cases }\end{array}$ & Low & High & P-value \\
\hline Age (years) & & & & 0.524 \\
\hline$\leq 50$ & 251 & 197 & 54 & \\
\hline$>50$ & 557 & 448 & 109 & \\
\hline Sex & & & & 0.606 \\
\hline Female & 802 & 639 & 163 & \\
\hline Male & 6 & 6 & 0 & \\
\hline Tumor stage & & & & 0.105 \\
\hline T1-T2 & 687 & 555 & 132 & \\
\hline T3-T4 & 121 & 90 & 31 & \\
\hline Lymph node stage & & & & $0.026^{\mathrm{a}}$ \\
\hline No & 385 & 320 & 65 & \\
\hline N1-NX & 423 & 325 & 98 & \\
\hline Metastasis stage & & & & $<0.0001^{\mathrm{b}}$ \\
\hline M0 & 688 & 567 & 121 & \\
\hline M1-MX & 120 & 78 & 42 & \\
\hline Stage & & & & 0.413 \\
\hline I-II (Tis) & 605 & 487 & 118 & \\
\hline III-IV & 203 & 158 & 45 & \\
\hline ER status & & & & 0.162 \\
\hline+ & 598 & 470 & 128 & \\
\hline- & 210 & 175 & 35 & \\
\hline PR status & & & & $0.044^{\mathrm{a}}$ \\
\hline+ & 519 & 403 & 116 & \\
\hline- & 289 & 242 & 47 & \\
\hline Her2 status & & & & $0.014^{\mathrm{a}}$ \\
\hline+ & 107 & 95 & 12 & \\
\hline- & 701 & 550 & 151 & \\
\hline
\end{tabular}

'The data were analyzed using Chi-square or Fisher's exact tests. ${ }^{\mathrm{a}} \mathrm{P}<0.05,{ }^{\mathrm{b}} \mathrm{P}<0.0001$. ER, estrogen receptor; $\mathrm{PR}$, progesterone receptor.

miR-204 on the proliferation of MCF-7 cells, we performed MTT assays. As shown in Fig. 2A, we confirmed that the cells in the LV-hsa-mir-204 group showed significantly inhibited growth in a time-dependent manner, with a particularly strong inhibitory effect being observed in the LV-hsa-mir-204 group at $96 \mathrm{~h}(0.63 \pm 0.01)$ when their growth was similar to that of the control $(0.88 \pm 0.03)$ and $\mathrm{NC}$ group $(0.86 \pm 0.04)$. Meanwhile, we also assessed apoptosis rates using flow cytometry. The LV-hsa-mir-204 group showed a much higher rate of apoptosis $(34.7 \pm 1.9 \%)$ than the control and NC groups (Fig. 2B).

miR-204 suppresses cell invasion and migration. We performed Transwell assays to explore whether miR-204 affects cell invasion and migration. Transwell invasion assays performed in Matrigel showed that the ability of MCF-7 cells to invade was inhibited in the LV-hsa-mir-204 group, and the number of cells that were transfected with the
Table II. Relationships between FOXA1 and clinicopathological parameters in the breast cancer cases $(\mathrm{N}=999)$.

\begin{tabular}{|c|c|c|c|c|}
\hline Characteristics & $\begin{array}{c}\text { No. of } \\
\text { cases }\end{array}$ & Low & High & $\mathrm{P}$-value ${ }^{\mathrm{a}}$ \\
\hline Age (years) & & & & $<0.0001^{\mathrm{b}}$ \\
\hline$\leq 50$ & 299 & 172 & 127 & \\
\hline$>50$ & 700 & 299 & 401 & \\
\hline Sex & & & & 0.349 \\
\hline Female & 989 & 468 & 521 & \\
\hline Male & 10 & 3 & 7 & \\
\hline Tumor stage & & & & 0.716 \\
\hline $\mathrm{T} 1-\mathrm{T} 2$ & 844 & 400 & 444 & \\
\hline T3-T4 & 155 & 71 & 84 & \\
\hline Lymph node stage & & & & $0.022^{\mathrm{a}}$ \\
\hline No & 475 & 242 & 233 & \\
\hline $\mathrm{N} 1-\mathrm{NX}$ & 524 & 229 & 295 & \\
\hline Metastasis stage & & & & 0.526 \\
\hline M0 & 860 & 402 & 458 & \\
\hline M1-MX & 139 & 69 & 70 & \\
\hline Stage & & & & 0.974 \\
\hline I-II (Tis) & 744 & 351 & 393 & \\
\hline III-IV & 255 & 120 & 135 & \\
\hline ER status & & & & $<0.0001^{\mathrm{b}}$ \\
\hline+ & 735 & 272 & 463 & \\
\hline- & 264 & 199 & 65 & \\
\hline PR status & & & & $<0.0001^{\mathrm{b}}$ \\
\hline+ & 635 & 231 & 404 & \\
\hline- & 364 & 240 & 124 & \\
\hline Her2 status & & & & 0.658 \\
\hline+ & 150 & 68 & 82 & \\
\hline- & 849 & 403 & 446 & \\
\hline
\end{tabular}

${ }^{a}$ The data were analyzed using Chi-square or Fisher's exact tests. ${ }^{\mathrm{a}} \mathrm{P}<0.05,{ }^{\mathrm{b}} \mathrm{P}<0.0001$. FOXA1, Forkhead box A1.

miR-204-containing vector (75 \pm 9 ) penetrated the membrane

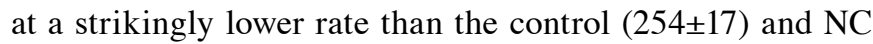
(242 \pm 22 ) cells (Fig. 3A, B and E). Transwell migration assays performed without Matrigel revealed that the number of cells that penetrated the membrane $(102 \pm 8)$ was clearly lower in the LV-hsa-mir-204 group than that in the control $(298 \pm 18)$ and NC (277 \pm 15$)$ groups (Fig. 3C, D and F).

miR-204 directly targets the 3'-UTR of FOXA1. To confirm that miR-204 directly targets FOXA1, potential binding sites were predicted using microRNA.org (Fig. 4A), and a luciferase report was subsequently performed (Fig. 4B). Luciferase activity was clearly lower in the cells that were co-transfected with FOXA1-WT 3'-UTR and miR-204 mimics (0.48 \pm 0.05$)$ than in the control cells, implying that miR-204 specifically binds to FOXA1 mRNA. Furthermore, the impact of miR-204 on the protein level of FOXA1 was assessed using western 

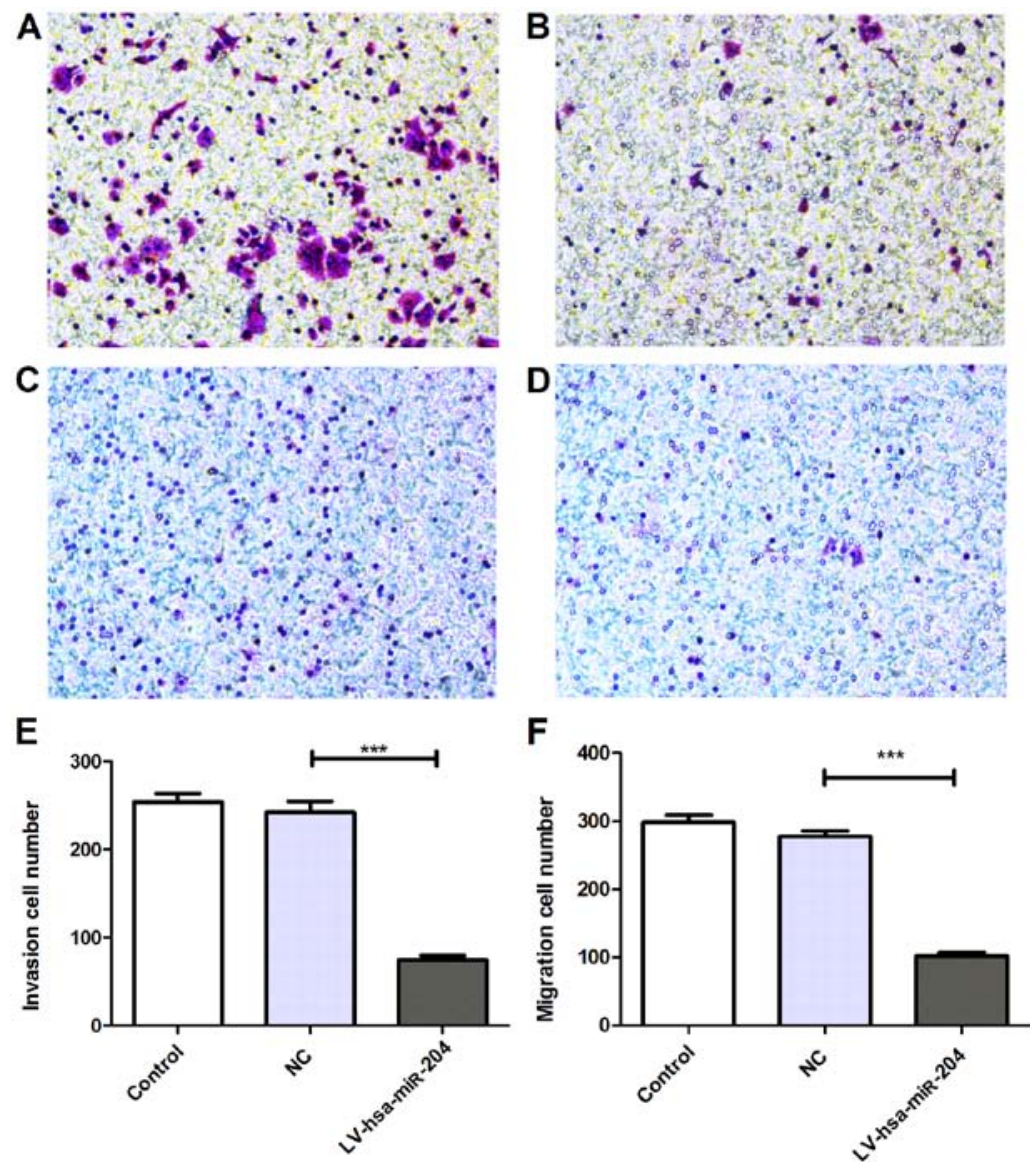

Figure 3. miR-204 inhibits the invasion and migration of breast cancer MCF-7 cells. (A, B and E) Transwell invasion assays were performed to evaluate miRNC- and miR-204-transfected cells. (C, D and F) Cells transfected with miR-NC and miR-204 were evaluated using Transwell migration assays. The cells was observed under an inverted microscope at a magnification of $\mathrm{x} 200 ;{ }^{* * *} \mathrm{P}<0.001$.

A

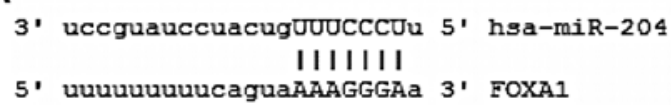

B

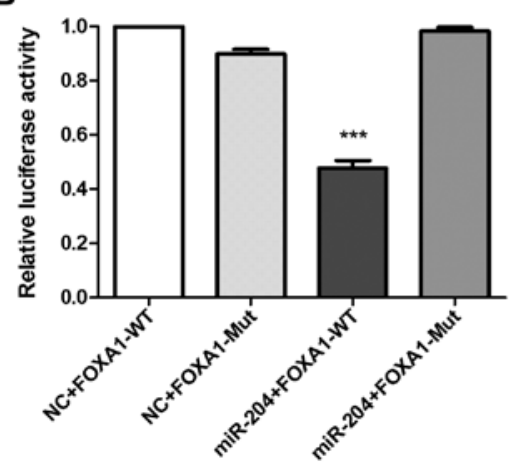

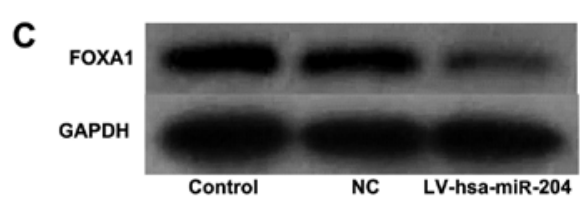

D

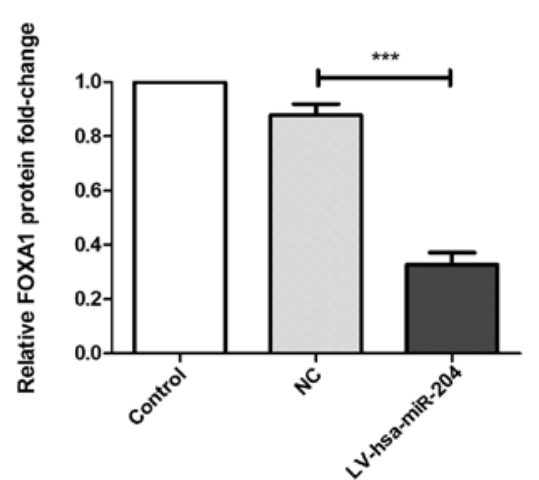

Figure 4. miR-204 directly targets the FOXA1 3'-UTR. (A) Potential complementary binding sites between miR-204 and FOXA1. (B) Relative luciferase activity was assessed after cells were co-transfected with FOXA1 and miR-204. (C) MCF-7 cells were transfected with miR-204-up and then subjected to western blot analysis. (D) The relative expression of FOXA1 protein was evaluated; ${ }^{* * *} \mathrm{P}<0.001$.

blot analysis. The results showed that miR-204-up clearly suppressed the protein level of FOXA1 (Fig. 4C and D). This change in the expression of the FOXA1 protein supports our hypothesis. 


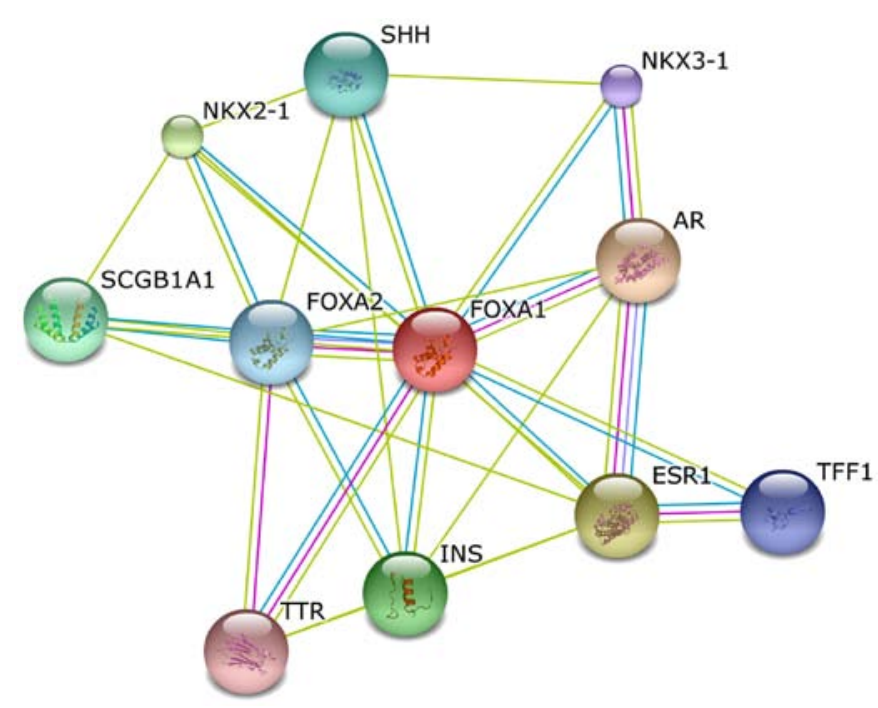

Figure 5. The FOXA1 network. Ten predicted functional partners were found to be related with FOXA1, and different colored lines represent evidence from different tests.

The FOXA1 network. Using the STRING database, we preliminarily characterized the FOXA1 network and identified 10 predicted functional partners, including androgen receptor (AR), estrogen receptor 1 (ESR1), NK2 homeobox-1 (NKX2-1), insulin (INS), secretoglobin, family 1A, member 1 (SCGB1A1), sonic Hedgehog (SHH), Forkhead box A2 (FOXA2), trefoil factor 1 (TFF1), NK3 homeobox-1 (NKX3-1) and transthyretin (TTR). Among these, the correlation scores for AR and ESR1 were the highest. All of the genes were tested using different experiments (Fig. 5).

\section{Discussion}

Breast cancer is a leading cause of cancer-related mortality in women. While methods for the diagnosis and systematical treatment of breast cancer have advanced, the prognosis for patients with this condition remains poor (29). In recent years, a large number of studies have reported that miRNAs are dysregulated in carcinomas via a series of biological processes. This influences the development of tumors by affecting processes including cell proliferation, apoptosis, the cell cycle and invasion and migration. Iorio et al (7) showed that miRNAs play a vital role in breast cancer by analyzing the structural characteristics and their functions in vivo. miR-204 was first identified as an anti-oncogene, and it was reported to downregulate non-small cell lung cancer (30), glioma (31), gastric (32) and thyroid cancer (33). Li et al (15) used RT-qPCR to show that the level of miR-204 was lower in breast cancer tissues than in adjacent normal breast tissues in 39 patients. In addition, lower levels of expression of miR-204 were associated with late TNM stage, distant metastasis and poor outcomes in a cohort of 129 breast cancer tissues (15). Wang et al (16) demonstrated that miR-204 targets JAK2 to regulate the STAT3/Bcl-2/survivin pathway in breast cancer. However, the potential molecular mechanism of miR-204 underlying its regulation of breast cancer progression is still ambiguous. Hence, we investigated the role of miR-204 by a series of in vitro experiments in the breast cancer cell line MCF-7.

As the number of samples used in previous studies was low, we consolidated relevant chips that were available in the TCGA database, and obtained relatively reliable results in the summary analysis. A low level of expression of miR-204 was associated with tumor stage and metastasis, and our preliminary results suggested that miR-204 may be involved in the process of metastasis in breast cancer. To further examine the effects of miR-204 on the biological function of breast cancer cells, a lentivirus that overexpressed miR-204 was transfected into MCF-7 cells. The results of MTT assays revealed that proliferation was markedly inhibited in the LV-hsa-mir-204 group of MCF-7 cells. Flow cytometry showed that the apoptosis rate was increased in the LV-hsa-mir-204 group. These results demonstrated that miR-204 restrained cell growth and induced apoptosis, which is in line with the results described by Wang et al (16). However, the difference was that we transfected a lentivirus rather than use chemical mimics, and we observed a higher transfection efficiency. The effect of transfection was therefore clearer, and the results were more reliable. A few studies have examined the role of miR-204 in invasion and metastasis in breast cancer, and we verified that cells markedly overexpressing miR-204 presented a reduced capacity to migrate into and invade the outer chamber in cell invasion and migration assays, similar to the results observed in glioma (31). miR-204 was reported to be downregulated in glioma tissues and to suppress glioma cell growth, invasion and metastasis by directly targeting RAB22A. In summary, these results indicate that miR-204 acts as a potential tumorsuppressor. The level of miR-204 was directly related to the progression of cancers, and its levels predicted the growth of the tumor, the prognosis, and even quality of life. In contrast, as miRNAs do not encode proteins, it is necessary for them to combine with hundreds of mRNAs to achieve their post transcriptional regulatory functions. We therefore attempted to identify potential target genes of miR-204 using online web tools, and 8,312 target genes were predicted. Some of these target genes have been studied. A functional analysis affirmed that miR-204 targets Bcl-2 and thereby acts as a tumor suppressor in gastric cancer (32). miR-204 also regulates HMGA2 to inhibit cell growth in human thyroid cancer (33). Among the nearly 8,500 genes that were identified, FOXA1 attracted our attention.

FOXA1 has been identified to participate in the formation and development of tumors, such as gastric (26), prostate (27), breast (28) and endometrial cancer (34). Knockdown of FOXA1 was found to inhibit proliferation and induce apoptosis in breast cancer MCF-7 cells (28). Importantly, FOXA1 is closely related to the estrogen receptor (ER), which plays a crucial role in breast cancer and the expression of which is correlated with ER-positive breast cancer (35). Mehta et al (36) reported that FOXA1 was upregulated in breast cancer. The expression of FOXA1 was positively correlated with ER-positivity and a good prognosis and was negatively correlated with tumor grade, tumor size, nodal status and HER2 levels $(36,37)$. In GOBO (http://co.bmc.lu.se/gobo/), samples from a total of 1,881 patients were statistically correlated across 11 types of data. The statistically analyzed results of 1,620 cases showed that FOXA1 was highly expressed in ER-positive breast cancer 
and that high levels of expression of FOXA1 were inversely proportional to their grade, where a higher grade was associated with lower FOXA1 expression. Therefore, patients with a high level of FOXA1 were found to have a better prognosis. We also obtained data related to cell lines from GOBO, which indicated that FOXA1 was strongly expressed in the MCF-7 cell line. Partial results related to FOXA1 for the tissues and chips were obtained from the GEO database (http://www. ncbi.nlm.nih.gov/geo/). The TCGA study identified FOXA1 as a key regulator of ER function in invasive lobular breast carcinoma, and the cBioPortal database showed that FOXA1 was altered in 25 (2\%) of 1,100 breast cancer samples. In all 25 of these cases, its expression was upregulated and the patients experience a poor outcome. A high level of FOXA1 in breast cancer was also correlated with lymph node stage, which may explained its essential role in the development of breast cancer. The related function network in cBioPortal, which we used to identify important tumor markers, showed that p53 and AFP play important roles in the evolution of cancer. In the STRING database, FOXA1 expression was predicted to share the strongest relationship with AR and ESR1, and it has been previously shown that both of these hormones are associated with mortality in females. Changes in their levels could result in the occurrence of various hormone-dependent diseases. Advanced age could also influence changes in the expression of AR and ESR1. FOXA1 may also influence AR and ESR1 through an interaction network. High FOXA1 levels were associated with age and were also indirectly shown to play a role in regulating hormone levels in breast cancer.

In the present study, we found that increased levels of miR-204 were associated with lower levels of FOXA1 at the mRNA level. As expected, western blot analysis revealed a negative correlation between FOXA1 and miR-204 levels. Thus, the focus of the present study was to investigate the functional mechanisms involved in the association between miR-204 and FOXA1. In luciferase reporter assays, the expression of a FOXA1 3'-UTR-containing plasmid was repressed in the presence of miR-204, while mutated FOXA1 3'-UTR resisted this effect. FOXA1 was also identified as a direct target of miR-204 in breast cancer MCF-7 cells. It is noteworthy that miRNAs can bind to multiple target genes to affect tumors. As previously mentioned, miR-204 regulated downstream factors by combining with Bcl-2 (16), and notably, Song et al (38) showed that Bcl-2 can specifically bind to FOXA1. Hence, a circular cycle may exist between miR-204, FOXA1 and Bcl-2, and they may jointly regulate apoptosis via the STAT3/Bcl-2/survivin pathway. miR-204 also regulates the function of tumors by altering the functions of these genes, which is supported by the present study of FOXA1. Nevertheless, many aspects of this interaction remain unexplored, and many experiments are needed to verify the hypothesis proposed in the present study.

The present study has some limitations. Firstly, the data were from experiments involving cell lines in vitro and lacked findings involving tissues. Results of experiments involving tissues could be further required from all the databases we searched with confirmation by RT-qPCR, immunohistochemical method and western blotting. Secondly, the functions and mechanisms proposed by the present study were explored and tested using only one cell line. Thirdly, in vivo experimental data were lacking.
In summary, we confirmed that miR-204 is downregulated in the MCF-7 cell line and suppressed cell growth, invasion and metastasis while promoting and stimulating apoptosis. FOXA1, as a direct target of miR-204, was found to be involved in the transcriptional control of downstream factors and pathways when it binds with miR-204. Therefore, miR-204 may be a novel predictive marker and a potential therapeutic target for breast cancer.

\section{Acknowledgements}

The present study was supported by grants from the National Natural Scientific Research Fund (nos. 81560386 and 81560489), and the Guangxi Natural Science Foundation (no. 2015jjAA40315).

\section{References}

1. Torre LA, Bray F, Siegel RL, Ferlay J, Lortet-Tieulent J and Jemal A: Global cancer statistics, 2012. CA Cancer J Clin 65: 87-108, 2015.

2. World Health Organization: IAfRoC: GLOBOCAN 2012. http://globocan.iarc.fr/Default.aspx. Accessed 08, 18, 2016.

3. Siegel RL, Miller KD and Jemal A: Cancer statistics, 2016. CA Cancer J Clin 66: 7-30, 2016.

4. Chen W, Zheng R, Baade PD, Zhang S, Zeng H, Bray F, Jemal A, Yu XQ and He J: Cancer statistics in China, 2015. CA Cancer J Clin 66: 115-132, 2016.

5. Di Leva G, Garofalo M and Croce CM: MicroRNAs in cancer. Annu Rev Pathol 9: 287-314, 2014

6. Hausser $J$ and Zavolan M: Identification and consequences of miRNA-target interactions - beyond repression of gene expression. Nat Rev Genet 15: 599-612, 2014.

7. Iorio MV, Casalini P, Piovan C, Braccioli L and Tagliabue E: Breast cancer and microRNAs: Therapeutic impact. Breast 20 (Suppl 3): S63-S70, 2011.

8. Huang Q, Gumireddy K, Schrier M, le Sage C, Nagel R, Nair S, Egan DA, Li A, Huang G, Klein-Szanto AJ, et al: The microRNAs miR-373 and miR-520c promote tumour invasion and metastasis. Nat Cell Biol 10: 202-210, 2008.

9. Chen J, Wang BC and Tang JH: Clinical significance of microRNA-155 expression in human breast cancer. J Surg Oncol 106: 260-266, 2012.

10. Dinami R, Ercolani C, Petti E, Piazza S, Ciani Y, Sestito R, Sacconi A, Biagioni F, le Sage C, Agami R, et al: miR-155 drives telomere fragility in human breast cancer by targeting TRF1. Cancer Res 74: 4145-4156, 2014.

11. Mackiewicz M, Huppi K, Pitt JJ, Dorsey TH, Ambs S and Caplen NJ: Identification of the receptor tyrosine kinase $A X L$ in breast cancer as a target for the human $m i R-34 a$ microRNA. Breast Cancer Res Treat 130: 663-679, 2011.

12. Piovan C, Palmieri D, Di Leva G, Braccioli L, Casalini P, Nuovo G, Tortoreto M, Sasso M, Plantamura I, Triulzi T, et al: Oncosuppressive role of p53-induced miR-205 in triple negative breast cancer. Mol Oncol 6: 458-472, 2012.

13. Bai WD, Ye XM, Zhang MY, Zhu HY, Xi WJ, Huang X, Zhao J, Gu B, Zheng GX, Yang AG, et al: MiR-200c suppresses TGF- $\beta$ signaling and counteracts trastuzumab resistance and metastasis by targeting ZNF217 and ZEB1 in breast cancer. Int J Cancer 135: 1356-1368, 2014.

14. Fu J, Xu X, Kang L, Zhou L, Wang S, Lu J, Cheng L, Fan Z, Yuan B, Tian P, et al: miR-30a suppresses breast cancer cell proliferation and migration by targeting Eya2. Biochem Biophys Res Commun 445: 314-319, 2014.

15. Li W, Jin X, Zhang Q, Zhang G, Deng X and Ma L: Decreased expression of miR-204 is associated with poor prognosis in patients with breast cancer. Int J Clin Exp Pathol 7: 3287-3292, 2014.

16. Wang X, Qiu W, Zhang G, Xu S, Gao Q and Yang Z: MicroRNA-204 targets JAK2 in breast cancer and induces cell apoptosis through the STAT3/BCl-2/survivin pathway. Int J Clin Exp Pathol 8: 5017-5025, 2015. 
17. Costa RH, Grayson DR and Darnell JE Jr: Multiple hepatocyte-enriched nuclear factors function in the regulation of transthyretin and alpha 1-antitrypsin genes. Mol Cell Biol 9: $1415-1425,1989$.

18. Nucera C, Eeckhoute J, Finn S, Carroll JS, Ligon AH, Priolo C, Fadda G, Toner M, Sheils O, Attard M, et al: FOXA1 is a potential oncogene in anaplastic thyroid carcinoma. Clin Cancer Res 15: 3680-3689, 2009.

19. Sano M, Aoyagi K, Takahashi H, Kawamura T, Mabuchi T, Igaki $\mathrm{H}$, Tachimori $\mathrm{Y}$, Kato H, Ochiai A, Honda H, et al: Forkhead box A1 transcriptional pathway in KRT7-expressing esophageal squamous cell carcinomas with extensive lymph node metastasis. Int J Oncol 36: 321-330, 2010.

20. Deutsch L, Wrage M, Koops S, Glatzel M, Uzunoglu FG, Kutup A, Hinsch A, Sauter G, Izbicki JR, Pantel K, et al: Opposite roles of FOXA1 and NKX2-1 in lung cancer progression. Genes Chromosomes Cancer 51: 618-629, 2012.

21. Gerhardt J, Montani M, Wild P, Beer M, Huber F, Hermanns T, Müntener $M$ and Kristiansen G: FOXA1 promotes tumor progression in prostate cancer and represents a novel hallmark of castration-resistant prostate cancer. Am J Pathol 180: 848-861, 2012.

22. Xu C, Wei Q, Guo J, Zhou JC, Mei J, Jiang ZN, Shen JG and Wang LB: FOXA1 expression significantly predict response to chemotherapy in estrogen receptor-positive breast cancer patients. Ann Surg Oncol 22: 2034-2039, 2015.

23. Huang Z, Huang L, Shen S, Li J, Lu H, Mo W, Dang Y, Luo D, Chen G and Feng Z: Sp1 cooperates with Sp3 to upregulate MALAT1 expression in human hepatocellular carcinoma. Oncol Rep 34: 2403-2412, 2015.

24. Tu H, Wei G, Cai Q, Chen X, Sun Z, Cheng C, Zhang L, Feng Y, Zhou H, Zhou B, et al: MicroRNA-212 inhibits hepatocellular carcinoma cell proliferation and induces apoptosis by targeting FOXA1. Onco Targets Ther 8: 2227-2235, 2015.

25. Chen G, Kronenberger P, Teugels E, Umelo IA and De Grève J: Targeting the epidermal growth factor receptor in non-small cell lung cancer cells: The effect of combining RNA interference with tyrosine kinase inhibitors or cetuximab. BMC Med 10: 28, 2012.

26. Ren H, Zhang P, Tang Y, Wu M and Zhang W: Forkhead box protein A1 is a prognostic predictor and promotes tumor growth of gastric cancer. Onco Targets Ther 8: 3029-3039, 2015.

27. Zhang C, Wang L, Wu D, Chen H, Chen Z, Thomas-Ahner JM, Zynger DL, Eeckhoute J, Yu J, Luo J, et al: Definition of a FoxA1 cistrome that is crucial for $\mathrm{G}_{1}$ to $\mathrm{S}$-phase cell-cycle transit in castration-resistant prostate cancer. Cancer Res 71: 6738-6748, 2011.
28. Zheng L, Qian B, Tian D, Tang T, Wan S, Wang L, Zhu L and Geng X: FOXA1 positively regulates gene expression by changing gene methylation status in human breast cancer MCF-7 cells. Int J Clin Exp Pathol 8: 96-106, 2015.

29. DeSantis C, Ma J, Bryan L and Jemal A: Breast cancer statistics, 2013. CA Cancer J Clin 64: 52-62, 2014

30. Guo W, Zhang Y, Zhang Y, Shi Y, Xi J, Fan H and Xu S: Decreased expression of miR-204 in plasma is associated with a poor prognosis in patients with non-small cell lung cancer. Int $\mathbf{J}$ Mol Med 36: 1720-1726, 2015.

31. Xia Z, Liu F, Zhang J and Liu L: Decreased expression of miRNA-204-5p contributes to glioma progression and promotes glioma cell growth, migration and invasion. PLoS One 10: e0132399, 2015.

32. Sacconi A, Biagioni F, Canu V, Mori F, Di Benedetto A, Lorenzon L, Ercolani C, Di Agostino S, Cambria AM, Germoni S, et al: miR-204 targets Bcl-2 expression and enhances responsiveness of gastric cancer. Cell Death Dis 3: e423, 2012.

33. Wu ZY, Wang SM, Chen ZH, Huv SX, Huang K, Huang BJ, Du JL, Huang CM, Peng L, Jian ZX, et al: MiR-204 regulates HMGA2 expression and inhibits cell proliferation in human thyroid cancer. Cancer Biomark 15: 535-542, 2015.

34. Qiu M, Bao W, Wang J, Yang T, He X, Liao Y and Wan X: FOXA1 promotes tumor cell proliferation through AR involving the Notch pathway in endometrial cancer. BMC Cancer 14: 78, 2014.

35. Hu Q, Luo Z, Xu T, Zhang JY, Zhu Y, Chen WX, Zhong SL, Zhao JH and Tang JH: FOXA1: A promising prognostic marker in breast cancer. Asian Pac J Cancer Prev 15: 11-16, 2014.

36. Mehta RJ, Jain RK, Leung S, Choo J, Nielsen T, Huntsman D, Nakshatri H and Badve S: FOXA1 is an independent prognostic marker for ER-positive breast cancer. Breast Cancer Res Treat 131: 881-890, 2012

37. Hisamatsu Y, Tokunaga E, Yamashita N, Akiyoshi S, Okada S, Nakashima Y, Aishima S, Morita M, Kakeji Y and Maehara Y: Impact of FOXA1 expression on the prognosis of patients with hormone receptor-positive breast cancer. Ann Surg Oncol 19: $1145-1152,2012$

38. Song L, Wei X, Zhang B, Luo X, Liu J, Feng Y and Xiao X: Role of Foxa1 in regulation of bcl 2 expression during oxidativestress-induced apoptosis in A549 type II pneumocytes. Cell Stress Chaperones 14: 417-425, 2009. 